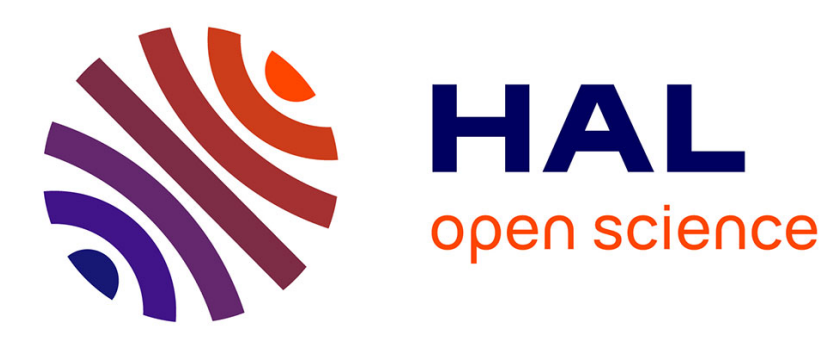

\title{
Le langage des anges. Charles Filiger et les instruments de sublimation magiques
}

Damien Delille

\section{To cite this version:}

Damien Delille. Le langage des anges. Charles Filiger et les instruments de sublimation magiques.

Histoire de l'art, 2013, 73, pp.159-168. halshs-01796698

\section{HAL Id: halshs-01796698 \\ https://shs.hal.science/halshs-01796698}

Submitted on 5 Jun 2018

HAL is a multi-disciplinary open access archive for the deposit and dissemination of scientific research documents, whether they are published or not. The documents may come from teaching and research institutions in France or abroad, or from public or private research centers.
L'archive ouverte pluridisciplinaire HAL, est destinée au dépôt et à la diffusion de documents scientifiques de niveau recherche, publiés ou non, émanant des établissements d'enseignement et de recherche français ou étrangers, des laboratoires publics ou privés. 
C'est au passage du $\mathrm{xx}^{\mathrm{e}}$ siècle qu'une nouvelle génération d'occultistes donne une connotation politique a l'androgyne angélique, avec l'histoire comparée des religions et l'étude scientifique ànes spirituels. Ces théories sont vulgarisées par Edouard sc 1889 des Grands Initiés ${ }^{10}$ démontrant que les fondateurs des religions monothéistes ont en commun l'idéal de réunion androgyne du masculin et du féminin. Schuré rappelle les rites de transformation d'Apollon à l'époque de Pythagore. L'adoration du soleil permet la fusion des genres sexuels : « ils concurent derrière ce feu sensible et cette lumière visible, un feu immatériel et une lumière intelligible. Ils identifièrent le premier avec le principe mâle, avec l'esprit créateur ou l'essence intellectuelle de l'univers, et la seconde avec son principe féminin, son âme formatrice, sa substance plastique ${ }^{11}$. La restauration de l'union originelle menée par l'idéal ésotérique lie science, art et religion, autour du culte solaire qui marque à terme les premières avant-gardes abstraites. L'art sacré fin-de-siècle se nourrit en retour de ces théories scientifiques de l'invisible qui permettent la restauration d'un homme nouveau androgyne.

L'idéalisme symboliste du critique d'art et écrivain Joséphin Péladan introduit l'image de l'androgyne mystique par le biais du primitivisme de la Renaissance. Les formes androgynes de la peinture italienne sont " le comble de l'art des formes réso[lu] à une fusion harmonieuse des deux sexes à leur puberté " ${ }^{12}$. Péladan publie en 1891 L'Androgyne avec le frontispice d'Alexandre Séon ${ }^{13}$ L'esquisse à la mine de plomb d'un jeune garçon de type florentin, à la chevelure bouclée et au regard hypnotique, représente l'idéal de neutralité sexuelle androgyne. La trame romanesque s'inspire de l'imaginaire romantique de Balzac et de Gautier ${ }^{14}$, en célébrant l'inversion des apparences sexuelles pour atteindre la neutralité des pôles opposés : "Jeune homme aux longs cheveux et presque désirable, que le désir n'a pas encore touché... Jeune fille aux courts cheveux et presque jouvenceau, dont le cœur n'est pas encore orienté " ${ }^{15}$. L'idéal de neutralité prôné par Péladan tente de "déjouer le paradigme ", ainsi que le définit Roland Barthes dans cette stratégie de rupture par l'union des opposés (femme/homme, bien/mal). « Le Neutre - mon Neutre - peut renvoyer à des états intenses, forts, inouis. 'Déjouer le paradigme' est une activité ardente, brûlante " ${ }^{16}$

La conception de neutralité de l'androgyne chrétien se retrouve sur les cimaises du premier salon de la Rose+Croix organisé par Péladan en 1892. Filiger présente La Prière ${ }^{17}$ (fig. 1), en hommage aux peintures sur bois des primitifs italiens. Sur un fond d'or de stvle byzantin, un adolescent prépubère coiffé d'une chevelure bleu de cobalt est agenouillé en prière. L'écrivain Rémy de Gourmont le compare aux travaux de "Cimabue, plus finis, plus poussés [...], un enfant en prière, adorable d'innocence " ${ }^{18}$. Les artistes invités par le mécène du salon et artiste Antoine de La Rochefoucauld privilégient les sources du primitivisme chrétien. Ce dernier décrit ainsi le Saint Jean-Baptiste ${ }^{19}$ (fig. 2)

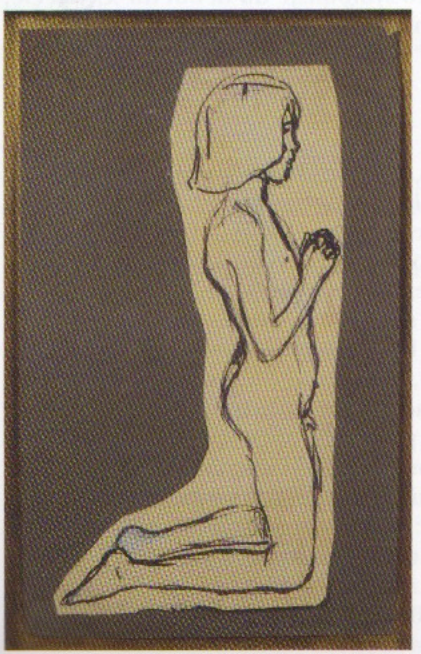

Charles Filiger, La Prière (esquisse), 1892, 34,5 x $21 \mathrm{~cm}$ Gent, Museum voor Schone Kunsten @ RES collection, Belgique.
Fig. 2. Charles Filiger, Saint Jean-Baptiste, vers 1892, 21,5 x 11,3 cm,

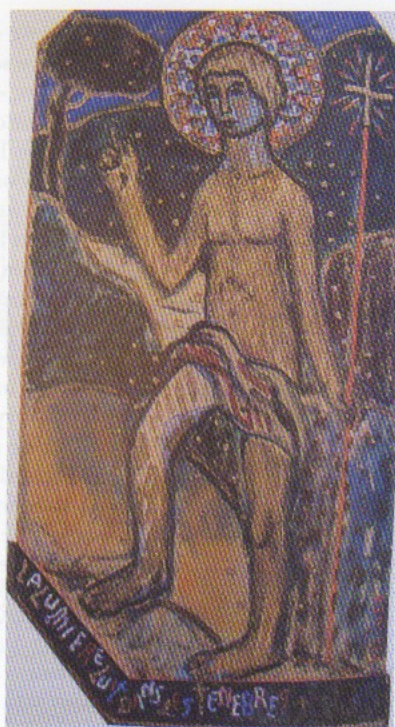

de Filiger: «que d'innocence, de noblesse, de candeur, de piété ! Vous aussi avez su retrouver le type 'androgyne', si cher aux vieux maîtres " ${ }^{20}$. L'article paru dans Le Cơur, revue fondée en 1893 par La Rochefoucauld et l'occultiste Jules Bois suite à la discorde avec Péladan, entérine deux visions de l'art idéaliste. Péladan ne supporte pas le primitivisme " déformateur ${ }^{21}$ de l'esthétique postimpressionniste à laquelle prend part Filiger. Afin de respecter l'ascèse religieuse visant au Grand Art classique, les règles du salon rosicrucien dictées par Péladan se radicalisent l'année suivante : exclusion des artistes femmes et des genres picturaux considérés comme inférieurs à l'art sacré. L'artiste, prêtre de l'idéal, doit respecter le célibat pour entrer en art comme on entre en religion. Admiratif de ce monitoire, Filiger adopte progressivement cette vie d'ascèse en devenant un " moine raté " selon les mots de La Rochefoucauld. Il part à partir de 1890 en Bretagne rejoindre le phalanstère de Pont-Aven formé par Gauguin et y trouve un remède à ses angoisses mystiques, suite à son départ hors de Paris après une rixe pour relation homosexuelle ${ }^{22}$

L'écrivain Alfred Jarry cerne le premier le trouble " mystico-sexuel » de Filiger dans son article de 1894. L'angélisme androgyne se retrouve dans la peinture auréolée de motifs décoratifs médiévaux, qui présente trois garçons entourant Sainte-Cécile ${ }^{23}$. De même, Le Lion et Saint Marc ${ }^{24}$ est selon Jarry une "peinture de moine avec des couleurs venues en écaille de pays étranges, étendues respectueusement aux sarcophages ${ }^{25}$. La description de l'Homme nu assis devant un paysage $e^{26}$ et des esquisses de jeunes " bretons résignés " retiennent l'attention de l'écrivain. Filiger les convertit en figures pieuses vouées à l'éternité : « Ne faites jamais ni pleurer ni rire vos figures " ${ }^{27}$, rappelle Jarry en reprenant les propos de l'artiste. La simplicité des figures, traitées selon le modèle synthétique de l'aplat et des lignes qui encerclent les formes, incarne landrogyne primitif Issue dur mythe platonicien ${ }^{28}$ l'image de la sphère androgyne, qui unifie le masculin et le féminin avant sa division ultime, renvoie à l'innocente pureté des anges. Selon Jarry, « de la synthèse du complexe se refait la simplicité première (Filiger, Bernard...), uniprimauté qui contient tout, comme l'un insexué engendre tous les nombres, portraiturant de chaque objet au lieu de la vie l'être, ou synonymes, le principe de synthèse (incarné particulier), l'idée ou Dieu ${ }^{29}$

L'idéal sphérique de Jarry s'inspire, au-delà de la référence platonicienne, des écrits du psychophysicien allemand Gustav-Theodor Fechner, allias « Dr Misès [qui] avait défini les anges ${ }^{30}$. Marqué par l'ésotérisme de Swedenborg, Fechner définit en 1825 dans son Anatomie comparée des anges l'idéal de communion colorée des sens par l'analogie des sphères amoureuses. Filiger serait guidé par la recherche harmonieuse de la synesthésie produite par les anges qui peuvent « communiquer leurs 
pensées par la lumière. En guise de sons, ils ont des couleurs ${ }^{31}$. La forme sphérique du soleil et l'unité primordiale de l'ange trouve son analogie dans la perception optique de l'homme. À l'image des romantiques, Fechner considère « l'œil [...] comme une créature solaire sur terre. Il vit dans et par les rayons du soleil, et présente donc la forme de ses frères du soleil lui-même. [...] En revanche, les créatures solaires que j'appelle les anges, en raison de leur nature supérieure sont des yeux devenus libres " " 2 . À la suite des théories optiques de Goethe, l'observation aveuglante du soleil par William Turner permet de comprendre en quoi, selon Jonathan Crary, le dépassement des limites sensorielles dans l'observation de la lumière conduit à un art non-mimétique ${ }^{33}$ Cette fusion ditre de l'œil et du soleil resurgit dans la peinture de Filiger, avec l'usage en aplat de couleurs pures représentant les figures de jeunes garçons angéliques.

La lumière, langage silencieux des anges, devient un guide spirituel pour neutraliser les affection considérées comme négatives. À la recherche des formes perdues de l'androgyne asexué, l'écrivain et l'artiste se retrouvent sur cette image sublimée d'amours homosexuelles interdites. La semiautobiographie Haldernablou de Jarry expose cet idéal mystico-sexuel. Le maître Haldern assassine son page Ablou, après avoir tenté de coucher avec lui. Sur fond de destruction biblique de Sodome, l'attraction sexuelle impossible conduit à l'asexualité. Cet amour crypté angélique, sûrement celui de Léon-Paul Fargue et Jarry lui-même, permet de sortir de la condamnation de l'homosexualité " Hors du sexe $[\ldots]$ ni homme, ni femme, ni tout à faire monstre, esclave dévoué et qui put parler sans rompre l'harmonie de mes pensées sublimes [...]. Quel homme t'a-t-il dit qu'il t'aimait, Ablou ? " ${ }^{34}$ Seule l'ascèse offre une vision purifiée qui donne à voir à terme le passé et l'avenir des

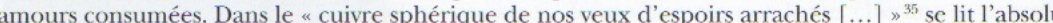
artistique, dont les formes lumineuses servent à communiquer à travers différentes temporalités.

\section{Les Notations Chromatiques el la recherche du "genre absolu "}

Cette recherche d'absolu par l'ascétisme sexuel se retrouve au cœur des relations tumultueuse entre Filiger et le peintre néerlandais Jan Verkade. Dès son arrivée en Bretagne en juin 1891, Verkade est initié par Filiger à la spiritualité, à travers les lectures de Schuré et de Balzac. Cette influence se retrouve dans la peinture intitulée Saint Sébastien ${ }^{36}$, inspirée des figures angéliques en aplat de Filiger. Dans les mémoires de Verkade, Filiger est surnommé Drahtmann, en allemand homme de fil, " à la nature très complexe " et à qui "seule la religion [...] apporte la grâce et l'aide surnaturelle " ${ }^{37}$ Verkade mentionne un épisode coïncidant avec le désir de se faire baptiser, ce qui entraîne son départ de Bretagne : «Quelque chose de grave faillit arriver. Un jour que nous étions plongés dans une conversation profonde, la tentation vint à nous, mais elle était trop balourde, notre sentiment esthétique en fut blessé, ce qui nous sauva ${ }^{38}$. Cette allusion cryptée aux désirs refoulés entre hommes se retrouve bien plus tard dans les correspondances du peintre Armand Seguin. Ordonné bénédictin à l'abbaye de Beuron en 1902, Verkade invite Seguin à se méfier de Filiger devenu, explique-t-il, « un terrible instrument dans les mains de Satan, un vrai loup dans des vêtements d'agneau » ${ }^{39}$ et à prier pour lui. aux expériences esthétiques des saintes mesures du père Desiderius Lenz (fig. 3), par la mise au carreau et la géométrisation des figures, "le tout tracé au compas et à la règle " ${ }^{41}$. Dès son retour en Hollande au début de l'année 1894, Verkade dit " prêcher à [s] es amis peintres l'évangile de la mesure du Père Desiderius ${ }^{42}$. Filiger est sûrement informé de ces expériences en $1894^{43}$, mai confirme surtout en 1903 connaître les activités des " Franciscains " et du grand " Hollandais devenu moine ${ }^{44}$. Son voyage en juillet 1903, pour voir son frère à Bâle, lui permet certainemen de faire un détour par Beuron, tandis que Maurice Denis et Paul Sérusier s'y rendent en novembre de la même année ${ }^{45}$. La comparaison entre les essais de motifs décoratifs menés par Verkade et Filiger entre 1903 et 1905 atteste de ces rapports ${ }^{46}$. Filiger abandonne les figure byzantines courbes au profit de visages géométriques, à l'image de la Tête de Christ dans une auréole stylisée $e^{17}$ (fig. 4), proche du Bon pasteur ${ }^{18}$ de Verkade (fig. 5). Tandis que ce dernier choisit l'ascèse religieuse, Filiger purifie ses désirs homosexuels par l'abstraction des figures. Son errance en Bretagne à partir de 1903 le conduit à être interné en octobre 1905 à l'hospice de Malestroit et à séjourner chez les trappistes de Thymadeuc en 1906. Se recueillant dans la solitude, il conçoit de nouvelles compositions non plus guidées « selon Dieu mais selon la Folie », associant la Bretagne à « un pays de magie ", où " il était nécessaire que mon Moi fut [sic] annihilé " ${ }^{9}$.

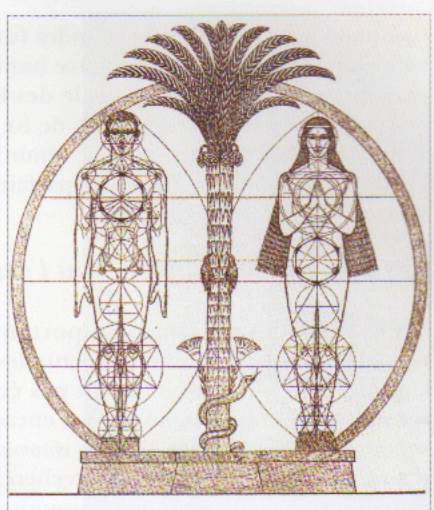

Fig. 3. Desiderius Lenz, Adam et Eve, $47,7 \times 39,2 \mathrm{~cm}$, Beuron, abbave Saint-Martin.

Après cette période, la datation des œuvres devient incertaine. Intitulées Notations chromatiques à partir d'une étiquette marchande ultérieure ${ }^{50}$, ces compositions circulaires et géométriques vont comporter des notes manuscrites dans les marges. Dans L'Homme à la Croix ${ }^{51}$, Filiger explique l'ascèse à laquelle il s'astreint : "Ici le hasard m'a conduit à faire le contraire de ce que j'avais en idée - J'ai fait le côté esthétique que je devais négliger... La recherche esthétique n'est donc qu'accessoire en ce cas à moins d'imprévu prêtant à la chose et encore est-il tout facultatif et ad libitum $\aleph^{52}$. L'ascèse esthétique éloigne des plaisirs de la figure pour retrouver l'image géométrique de l'être primitif décrit par Lenz: « Le but de tout grand art est la transmission, l'application caractéristique des formes fondamentales géométriques [...]. L'homme lui-même, Adam, Dieu [... l'a tiré de la figure fondamentale du triangle, qui renferme le pair et l'impair, le masculin et le féminin, la division en deux et en trois selon l'expression de Kepler, 'l'homme-femme' "53.

La plupart des Notations chromatiques sont bifaces. La pauvreté de l'artiste explique cette économie de papier, qui révèle aussi la recherche de transparence obtenue par l'usage du papier à calquer. L'application des saintes mesures crée un système de grilles aquarellées de plus en plus complexe, par un enchevêtrement de lignes tracées formant un kaléidoscope coloré. La mise en confrontation
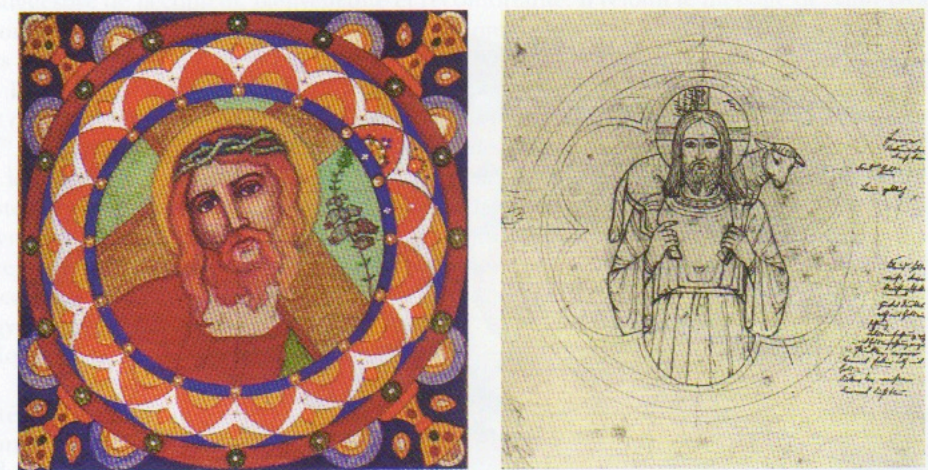

Fig. 4. Charles Filiger, Tềte de Chrisı dans une auréole stylisée, vers 1905, 17 x $17 \mathrm{~cm}$, Quimper, musée des Beaux-arts (c) Musée des Beaux-arts de Quimper. 
La dominante des couleurs chaudes (orange, jaune, rouge et rose) forme de nouveaux contrastes colorés par le jeu de transparence lumineuse. Chaque face se correspond à quelques millimètres pres, permettant la fusion ideale des figures, at image des anges de Fechmer premme cité par Lenz à la suie de Kepler. Dans une Notalion choulique, un achevée renvoie à l'autre figure fêminine inacheve, quicontiententigue, unc Filiger : « Le type ici est des plus parfaits dans le genre absolu $»^{55}$

\section{Les instruments magiques ou l'ascèse abstraite}

Les ouvrages de Lenz comportent de nombreux modèles de figures géométriques qui appellent les structures architecturales gothiques du fameux carnet de Villard de Honnecourt ${ }^{56}$ Ces motifs n'ont certainement pas échappé à Filiger, qui explique faire des « recherches de formules décoratives pour servir d'encadrement à [s] es petits sujets ${ }^{57}$, après sa visite au Louvre en septembre 1901. Dans la Notation chromatique dite Prométhée $e^{58}$ (fig. 6), Filiger signale que sa " méthode cristalline s'éloigne ainsi du cloisonnisme synthétique de Gauguin (selon les initiales «P. de G. ») Filiger recherche l'harmonie colorée par le biais d'un système de juxtapositions simultanées des contrastes, au moment même où Robert Delaunay développe le cubisme orphique à partir du contraste simultané des disques colorés. Ses figures deviennent de plus en plus abstraites et abandonnent toute distinction entre le féminin et le masculin.

La rencontre en 1909 de Filiger avec le musicien, peintre et maître verrier Richard Burgsthal ${ }^{60}$, par le biais d'Émile Schuffenecker, permet d'interpréter le fonctionnement magique des Notations chromatiques. Filiger envoie à Burgsthal des croquis de "morceaux d'enluminures» ${ }^{61}$ et " autres dessins ", qui vont probablement lui servir d'inspiration pour deux projets, la préparation des vitraux de Fontfroide commandés par Gustave Fayet en $1912^{62}$ et l'illustration de l'ouvrage posthume Les Clefs de l'Orient de l'occultiste Alexandre Saint- Yves d'Alveydre ${ }^{63}$. Burgsthal illustre une seule fois dans l'ouvrage les formes géométriques du double triangle dans le cercle issues du symbolisme théosophique (fig. 7). C'est sans doute à cette occasion que Filiger côtoie les théories ésotériques de Saint-Yves d'Alveydre, qui prône l'émancipation de la sexualité à travers l'image de l'androgyne "masculin-féminin " ${ }^{64}$, incarnation de l'union parfaite entre Dieu et la nature.

Les ouvrages de Saint-Yves d'Alveydre décrivent aussi l'usage de l'archéomètre, instrument censé lire le passé et l'avenir à partir de calculs complexes permettant de répondre aux mystères divins ${ }^{65}$. Proche du prognomètre de l'occultiste swedenborgien Josef Hoëné Wronski et s'inspirant de l'arithmosophie d'Athanasius Kircher, cet instrument fournit un alphabet universel liant les religions et les sens en un

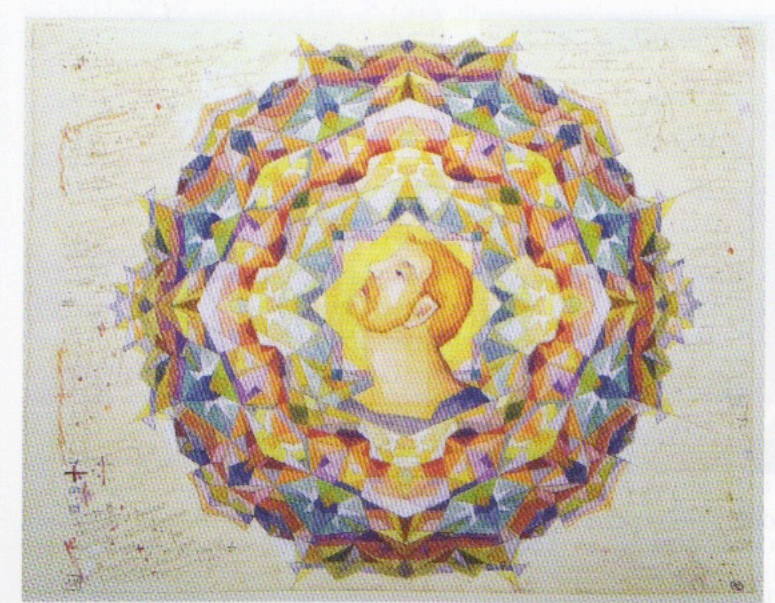

Fig. 7. Richard Burgsthal, «Ainsi l'arbre généalogique de la science », dans Alexandre Saint-Yves d'Alveydre, Les Clefs de l'Orient, 1910, Paris, (in

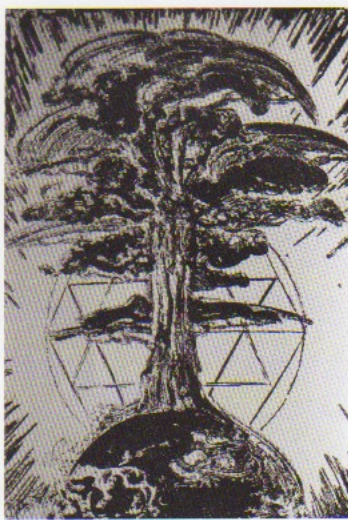

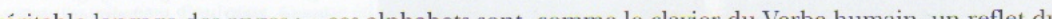
Verbe divin s'adressant à nos divers sens : par la Forme, par la Couleur, par le Son, et même par notre faculté intellectuelle la plus élevée, celle de l'Abstraction, par le Nombre "66. La combinaison des sens, liée aux signes du zodiaque et répartie à travers des couronnes circulaires, sert " l'œuvre maîtresse de l'Architechnie, l'édifice religieux ${ }^{67}$. Le but de l'archéomètre, instrument réalisant un art total par le biais de l'architecture religieuse, est de retranscrire « la langue angélique primordiale (celle des Correspondances de la parole et du Verbe) ${ }^{68}$, proche en ce sens du modèle des Notations chromatiques. La synthèse des arts présente dans les correspondances baudelairienne et wagnérienne donne à l'objet sacré un pouvoir cathartique recherché par Filiger. Dès 1901, il déclare se perdre « dans l'Unité même. Source de 'toute Harmonie'. [...] C'est par les voies les plus humaines, les plus basses, que je cherche la route qui conduit 'en haut' " ${ }^{69}$. La poursuite des rythmes musicaux guide son désir de purification décrit à sa nièce Anna : «la petite ceuvre est plus musicale que picturale, et qu'en cela je me réalise moi-même de ce que l'art a de consolant et de bienfaisant quand on le pratique à l'égal d'une religion et de la plus belle des religions, celle de l'âme $!{ }^{70}$ Ces œuvres servent d'objets expiatoires, lui renvoyant une « image du bon Dieu » dont il ne se sépare jamais, « l'aimant comme ma création la plus simple, comme le type le plus parfait de toutes mes autres figures " ${ }^{71}$. Tout en confiant à La Rochefoucauld le chemin qu'il s'est tracé, "par l'abstraction horrible qu' [il s'] efforce de suivre " ${ }^{72}$, Filiger tire partie de la puissance suggestive de la couleur, bienveillante et réconfortante. Il rejoint le message de Saint-Yves d'Alveydre, dont l'archéomètre prédit l'avènement d'un homme nouveau androgyne.

Les projets de vitraux de Burgsthal apportent une autre clé de compréhension aux Notations chromatiques. L'application musicale de l'archéomètre en fonction des signes astrologiques se retrouve tout d'abord dans les croquis préparatoires aux vitraux dessinés par Rita Strohl, la femme de Burgsthal ${ }^{73}$. Ces astres entourent des figures d'anges ailés de profil dans les vitraux de Fontfroide et rappellent les anges de la composition Le Jugement dernier de Filiger ${ }^{74}$ (fig. 8). Les rapports entre les deux artistes nécessitent encore néanmoins d'être éclaircis. Tous deux partagent la fascination de l'androgyne angélique, qui se matérialise dans la lumière colorée du vitrail, similaire au fonctionnement des Nolations chromatiques. Le vitrail devient un véritable langage sacré, lorsque le processus de confection se rapproche de l'alchimie et réalise l'union originelle des deux sexes en une entité androgync supérieure. Burgsthal rappelle ainsi : "C'est dans la partie supérieure que l'on met a Poêle ou Moufle, faite de bonne terre résistante au feu, Terre d'Adam mélangée de Terre d'Eve »

La postérité des Notations chromatiques est due à la signification hermétique de ces compositions produites, semble-t-il, à l'écart des premières avant-gardes cubiste et fauve. Filiger disparaît en effet des circuits artistiques et se trouve exposé de manière posthume au salon des Indépendants de 1914 Cependant, on retrouve des envois de panneaux décoratifs au même salon de $1920^{76}$, sûrement des Notations chromatiques tardives aux figures géométriques abstraites. La présence de ces compositions rappelle qu'une peinture figurative de jeune garçon de $1922^{77}$ fut sauvée d'un ensemble plus vaste, brûlé par les personnes qui l'avaient recueilli, « parce que [Filiger] peignait des petits garçons nus 
41. Verkade, Le Tourment de Diel, p. 251.

42. Ibid., p. 257.

43. La correspondance entre Verkade et Filiger se poursuit entre 1892 et 1894, années où Verkade est initié aux saintes mesures. Une rencontre a sûrement lieu en 1894 à Florence, citée dans C. Boyle-Turner, Jan Amsterdam, 1989), Zwolle Amsterdam, Waanders, Rijksmuseum Vincent van Gogh, 1989, note 2, p. 90, « information donnée par le Dr. Bogomila WelshOvcharov, provenant d'une source inédite ".

44. Lettre de Filiger à Séguin, musée départemental Maurice Denis Le Prieuré de Saint-Germain-n-Laye, fonds Charles Filiger, non folioté, non coté.

45. Rappelons que Sérusier traduit en français les écrits de Lenz, dans P. Lenz, L'Esthétique de Beuron, traduite de l'allemand par Paul Sérusier avec une introduction de Maurice Denis, Paris, Bibliothèque de l'Occident, 1905.

46. Il s'agit de panneaux décoratifs produits par Verkade pour le monastère bénédictin du mont Cassin et d'une série de croquis de Filiger, contenus dans le carnet dónen par Breton et vendu en 2003 au musée des Beaux-Arss de Quimper.

47. Charles Filiger, Tête de Christ dans une auréole stylisée, vers 1905 .

48. Jan Verkade, Bon pasteur, sans datc.

49. Lettre de Filiger à son frère Paul Filiger, octobre 1905 , Hospice de Malestroit, fonds Charles Filiger.

50. Voir l'étiquette de la figure 122, dans Jacob, Filiger linconnu, p. 91.

51. Charles Filiger, L'Homme à la cmix, sans date.

52. Reproduit dans Filiger: dessins, gouaches, aquarelles (cat. exposition : Saint-Germain-en-Laye, 1981), Saint-Germainen-Laye, musée départemental du Prieuré, 1981, p. 96.

53. Lenz, LEsthétique de Beuron, p. 18-19.

54. On remarquera que souvent l'une des faces es raturée d'une croix rouge, signalant les côtés pile et face. Je soutiendrais que ce n' est pas Filiger qui a produit ces croix, mais ses ayants droit, ayant voulu trouver un sens de lecture à ces notations et ayant choisi délibérement un côté, avec une couleur différente du crayon gris des note manuscrites de l'artiste.

55. Reproduit dans Jacob, Filiger l'inconnu, p. 190, notice 140. 56. Carnet de Villard de Honnecourt: d'après le manuscrit conserve a la Biblothéque nationale de Panis, $n^{\circ} 79093$, présenté Pernoul Jean Gimpel, Roland Bech Pes6. Voir non Gimpel, Roland Bechnan, Paris, Stock,

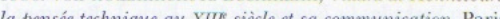

57. Lettre de Filiger à Schuffenecker reproduit dan « Lettre 5. novembre 1909 ", Charles Filiger. André Breton. la recherche de l'art magique, p. 70.

58. Charles Filiger, Notation chromatique dite Prométhée, sans date (vers 1910-15).

59. Notations chromatiques, sans date, dans Jacob, Filiger l'inconnu, p. 189, notice 140
60. Ce rapprochement n'a malheureusement pas été fai Burgsthal a été associé à un marchand d'art dans Charlós Filiger: André Breton. À la recherche de l'art magique, p. 70-73.

61. Ibid. Lors de ces échanges, Filiger lui envoie un "Christ agonisant entouré de têtes d'anges ", un maquette de "la Bete qui symbolise la Matière "et une Sainte Cécile, réalisant ainsi le " tout absolu - qui se peu exprimer par la ligne et la couleur

62. Voir à ce sujet M. Rougeot, Gustave Fayet (1865-1925) Itineraire d'un artiste collectionneur, thèse de doctorat sous la direction de Ségolène Le Men et de Rodolph Rapetti, Université Paris X (Nanterre), 2013.

63. A. Saint-Yves d'Alveydre, Les Clefs de l'Orient : les mystères de a naissance, les sexes et l'amour, les mystènes de la mort, d'après l Lefs de la Caboale on tatale, Paris, Librairie hermétique, 1910. 64. Ibid., p. 30-31.

i5. Voir à ce sujet B. Hapel, René Guénon el \& L'archéomètre Paris, G. Trédaniel, 1996 et J. Godwin, L'Ésotérisme musical

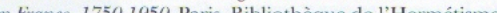
Albin Michel, 1991.

66. Charles Barlet, Saint-Yves d'Alveydre, p. 116

67. A. Saint-Yves d'Alveydre, L'Archéomètre, clef de toutes les religions el de toutes les sciences de l'antiguité réforme synthétigue de ious les arts contemporains, Paris, Dorbon âné, [1909], 146.

68. Ibid., p. 41.

69. Lettre de Filiger à La Rochefoucauld, 1901, fonds Charles Filiger.

70. Leture de Filiger à Anne Filiger, décembre 1904, fond Charles Filiger.

71. Lettre de Filiger à La Rochefoucauld, novembre 1899 fonds Charles Filiger:

72. Ibid.

73. Voir à ce sujet M. d'Angelo (éd.), La Musique à la Belle Epoque : autour du foyer artistique de Gustave Fayel, Béziers. Paris-Fontyroude, 1898-1914, Paris, Ed. Ic Manuscrit, 2013 et Richard Burgsthal, peintre el verner (1884-1944) (cal. de Béziers, 1999. Je remercie le musée de Béziers pou eurs informations au sujet de Burgstha.

74. Charles Filiger, Le Jugement dernier, 1898.

75. R. Burgsthal, Les Précieux Vitraux qui onnent ses fenêtres, Paris, Jean Naert, 1933, p. 45.

76. Trente ans d'art indépendant, 1884-1914: catalogue, xposition retrospective des auvres des membres inscrits au conis des trente premières expositions de la Société (cat. exposition Paris, 1920), [Paris], Société des artistes indépendants, 1914, p. 191 et 31' Exposition de la Société des artiste indépendants (cat. exposition : Paris, 1920), [Paris], Société des artistes indépendants, 1920 , p. 69 .

77. M. Jacob, Filiger lïnconnu, p. 115

78. Ibid., p. 150 .

79. Notations dans l'œuvre Le Neveu de Périclès, reproduite dans M. Jacob, Filiger linconnu, notice 181 , p. 199 